\title{
The Kalman-Yakubovich-Popov Lemma in a behavioural framework
}

\author{
Robert van der Geest ${ }^{\mathrm{a}, *}$, Harry Trentelman ${ }^{\mathrm{b}}$ \\ a Department of Applied Mathematics, University of Twente, P.O. Box 217, 7500 AE Enschede, Netherlands \\ b Mathematics Institute, University of Groningen, P.O. Box 800, 9700 AV Groningen, Netherlands
}

Received 20 January 1997; received in revised form 5 June 1997; accepted 28 June 1997

\begin{abstract}
The classical Kalman-Yakubovich-Popov Lemma provides a link between dissipativity of a system in state-space form and the solution to a linear: matrix inequality. In this paper we derive the KYP Lemma for linear systems described by higher-order differential equations. The result is an LMI in terms of the original coefficients in which the dissipativity problem is posed. Subsequently we stucly the connection between dissipativity and spectral factorization of polynomial matrices. This enables us to derive a new algorithm for polynomial spectral factorization in terms of an LMI in the coefficients of a polynomial matrix. (C) 1997 Elsevier Science B.V.
\end{abstract}

Keywords: Dissipative systems theory; Two-variable polynomial matrices; Linear matrix inequalities; Polynomial spectral factorization

\section{Introduction}

The Kalman-Yakubovich-Popov (KYP) Lemma is a classical result relating dissipativity of a system in state-space form to the existence of a solution to a linear matrix inequality (LMI). The KYP Lemma has always been recognized as a key result in systems theory, and over the last years it has regained popularity due to the availability of fast numerical routines for solving LMIs. A formulation and a proof of the classical KYP Lemma may be found in [6].

In this paper we formulate the KYP Lemma for linear differential systems, that is, systems described by a set of linear, higher-order differential equations involving the variables associated with the system. Such descriptions are commonly studied in the behavioural approach to linear systems theory, see for instance [7]. nl.

${ }^{*}$ Corresponding author. E-mail: r.a.b.vandergeest@math.utwente.
This paper is about systems that are dissipative with respect to a certain supply rate. This supply rate is specified in terms of quadratic differential forms (QDFs). Recently, Willems and Trentelman [8] introduced QDFs for specifying dissipativity in a behavioural framework. A QDF is a quadratic function of the variables associated with the system and some of their higher-order derivatives. Therefore, QDFs are particularly apt to specify quadratic expressions involving the variables of differential systems.

The line of reasoning in this paper is as follows. First, we formulate a KYP Lemma for generalized first-order systems, like in [1]. Then we transform the property of dissipativity in a higher-order framework into an equivalent property for a first-order system, in terms of the coefficients of the higher-order system. Plugging this first-order system into the KYP Lemma leads to an LMI in terms of the original coefficients. 
A system is dissipative with respect to a certain supply rate iff there exists a storage function, and this storage function is related to the solution to the LMI in the KYP Lemma. It may be found in [8] that an alternative way of finding storage functions is by performing a polynomial spectral factorization of a polynomial matrix associated with the supply rate. We use this connection in the last part of this paper to present a new algorithm for polynomial spectral factorization in terms of an LMI.

\section{Dissipativity in a behavioural framework}

This paper is about linear, time-invariant, differential systems, that is, linear, time-invariant, continuous-time systems, whose behaviour consists of all solutions $w$ to a linear differential equation of the form

$R\left(\frac{\mathrm{d}}{\mathrm{d} t}\right) w=0$.

Here $R \in \mathbb{R}^{p \times q}[\xi]$ is a $p \times q$ polynomial matrix in the indeterminate $\xi$ of a certain degree $N$,

$R(\xi):=R_{0}+R_{1} \xi+R_{2} \xi^{2}+\cdots+R_{N} \xi^{N}$.

A description of the form given by Eq. (1) is called an autoregressive representation, or kernel representation, and its properties are commonly studied in the behavioural approach to linear systems theory. (See for instance [7].) The behaviour described by Eq. (1) is defined as the set of all $w$ that satisfy the differential equation,

$\mathscr{B}:=\left\{w \in \mathscr{C}^{\infty}\left(\mathbb{R}, \mathbb{R}^{q}\right)\right.$ s.t. Eq. (1) $\}$.

Here $\mathscr{C}^{\infty}\left(\mathbb{R}, \mathbb{R}^{q}\right)$ is the set of infinitely often differentiable functions from $\mathbb{R}$ to $\mathbb{R}^{q}$. The system $\mathscr{B}$ represented by Eq. (1) is called controllable if for all $w_{1}, w_{2} \in \mathscr{B}$ there exists a $w \in \mathscr{B}$ and a $T \geqslant 0$ such that

$w(t)= \begin{cases}w_{1}(t) & \text { for } t<0, \\ w_{2}(t+T) & \text { for } t \geqslant 0 .\end{cases}$

The following result may be found in [7].

Lemma 2.1. The system $\mathscr{B}$ represented by Eq. (1) is controllable iff $R(\lambda)$ has the same rank for all $\lambda \in \mathbb{C}$.

The representation given by Eq. (1) is minimal if the matrix $R$ has full row-rank. It may be found in [7] that every differential system admits a minimal kernel representation. Moreover, two minimal kernel representations with polynomial matrices $R_{1}$ and $R_{2}$ represent the same behaviour iff there exists a unimodular matrix $U$ such that $R_{1}=U R_{2}$.

In [8] it is shown how quadratic expressions in the variables of differential systems are described very adequately using quadratic differential forms. Let $\Phi \in \mathbb{R}_{m}^{q \times q}[\zeta, \eta]$ be a symmetric two-variable polynomial matrix in the commuting indeterminates $\zeta$ and $\eta$,

$\Phi(\zeta, \eta):=\sum_{0 \leqslant i, j \leqslant K} \Phi_{i j} \zeta^{i} \eta^{j}$

where the matrices $\Phi_{i j} \in \mathbb{R}^{q \times q}$ satisfy $\Phi_{i j}=\Phi_{j i}^{\mathrm{T}}$, and where $K$ is the degree of $\Phi$, i.e., $K$ is the highest power of $\zeta$ and $\eta$ appearing in $\Phi(\zeta, \eta)$. Consider signals $w \in \mathscr{C}^{\infty}\left(\mathbb{R}, \mathbb{R}^{q}\right)$. The quadratic differential form (QDF) associated with $\Phi$ is

$Q_{\Phi}(w):=\sum_{0 \leqslant i, j \leqslant K}\left(\frac{\mathrm{d}^{i} w}{\mathrm{~d} t^{i}}\right)^{\mathrm{T}} \Phi_{i j}\left(\frac{\mathrm{d}^{j} w}{\mathrm{~d} t^{j}}\right)$.

The tilde " " " is used to denote the matrix containing the coefficients of a polynomial matrix or two-variable polynomial matrix. Thus, the coefficient matrix associated with $R$ is

$\tilde{R}:=\left(R_{0} R_{1} \cdots R_{N}\right)$

and the coefficient matrix associated with $\Phi$ is

$\tilde{\Phi}:=\left(\begin{array}{cccc}\Phi_{00} & \Phi_{01} & \cdots & \Phi_{0 K} \\ \Phi_{10} & \Phi_{11} & \cdots & \Phi_{1 K} \\ \vdots & \vdots & \ddots & \vdots \\ \Phi_{K 0} & \Phi_{K 1} & \cdots & \Phi_{K K}\end{array}\right)$.

Note that

$Q_{\Phi}(w)=\left(\begin{array}{c}w \\ \dot{w} \\ \ddot{w} \\ \vdots \\ w^{\left(K^{\prime}\right)}\end{array}\right)^{\mathrm{T}} \check{\Phi}\left(\begin{array}{c}w \\ \dot{w} \\ \ddot{w} \\ \vdots \\ w^{\left(K^{\prime}\right)}\end{array}\right)$.

Let $D\left(\mathbb{R}, \mathbb{R}^{q}\right)$ be the subset of $\mathscr{C}^{\infty}\left(\mathbb{R}, \mathbb{R}^{q}\right)$ consisting of functions that have compact support. The following result from [8] is that dissipativity of a system is equivalent to the existence of a storage function.

Lemma 2.2. Assume that the system 20 resented by Eq. (1) is controllable. Then the following two 
statements are equivalent:

(1) For all $w \in \mathscr{B} \cap D\left(\mathbb{R}, \mathbb{R}^{q}\right)$,

$\int_{-\infty}^{\infty} Q_{\Phi}(w)(t) \mathrm{d} t \geqslant 0$.

(2) There exists a symmetric two-variable polynomial matrix $\Psi$ such that

$\frac{\mathrm{d}}{\mathrm{d} t} Q_{\Psi}(w) \leqslant Q_{\Phi}(w)$ for all $w \in \mathscr{B}$.

Stated in words, the first statement in Lemma 2.2 says that the system $\mathscr{B}$ is dissipative with respect to the supply rate $Q_{\Phi}$, and the second statement in Lemma 2.2 says that the QDF $Q_{\Psi}$ is a storage function for the supply rate $Q_{\Phi}$ on the behaviour $\mathscr{B}$.

It turns out that storage functions are closely related to the state of the system. The notion of state of a differential system is studied by Rapisarda and Willems [4]. A latent variable representation of $\mathscr{B}$ with latent variable $x$ of the form

$$
\begin{array}{r}
\mathscr{B}=\left\{w \in \mathscr{C}^{\infty}\left(\mathbb{R}, \mathbb{R}^{q}\right) \mid \exists x \in \mathscr{C}^{0}\left(\mathbb{R}, \mathbb{R}^{q}\right)\right. \text { s.t. } \\
G w+F x+E \dot{x}=0\}
\end{array}
$$

is called a state representation of $\mathscr{B}$ with state variable $x$. We mean in Eq. (12) that the differential equation $G w+F x+E \dot{x}=0$ is satisfied in the sense of distributions. The full behaviour of such a state representation is defined as

$$
\begin{aligned}
\mathscr{B}_{\mathrm{f}}:=\left\{\left(\begin{array}{c}
w \\
x
\end{array}\right) \in \mathscr{C}^{\infty}\left(\mathbb{R}, \mathbb{R}^{q+n}\right)\right. \\
\text { s.t. } G w+F x+E \dot{x}=0\} .
\end{aligned}
$$

The following result from [5] says that if the twovariable polynomial matrix $\Phi$ is a constant matrix, then every storage function is a quadratic function of every state of the system.

Lemma 2.3. Let $\Phi \in \mathbb{R}^{q \times q}$. Let $Q_{\Psi}$ be a storage function for $Q_{\Phi}$ on the behaviour $\mathscr{B}$. Let $G w+F x+E \dot{x}=0$ be a state representation of the system $\mathscr{B}$ with full tehaviour $\mathscr{B}_{\mathrm{f}}$. Then there exists a symmetric matrix $K \in \mathbb{R}^{n \times n}$ such that

$Q_{\Psi}(w)=x^{\mathrm{T}} K x$ for all $\left(\begin{array}{c}w \\ x\end{array}\right) \in \mathscr{B}_{\mathrm{f}}$.

\section{The KYP Lemma, for first-order systems}

In this section we formulate the KYP Lemma for generalized first-order systems that are controllable and trim. This is the first step on the way to a KYP Lemma for differential systems: in the next section it turns out that a controllable differential system admits a generalized first-order representation that is controllable and trim. Consider a system in generalized first-order representation,

$G \dot{w}=F w$,

where $G$ and $F$ are real-valued, $p \times q$ matrices. Define the behaviour described by Eq. (15) as

$\mathscr{B}:=\left\{w \in \mathscr{C}^{\infty}\left(\mathbb{R},\left[\mathbb{R}^{q}\right)\right.\right.$ s.t. Eq. (15) $\}$.

The set of consistent points of Eq. (15) is

$W_{0}:=\left\{w_{0} \in \mathbb{R}^{q} \mid \exists w \in \mathscr{B}\right.$ s.t. $\left.w(0)=w_{0}\right\}$.

The representation given by Eq. (15) is called trim if $W_{0}=\mathbb{R}^{q}$.

The KYP Lemma for generalized first-order systems is formulated as follows.

Theorem 3.1. Let $M=M^{\mathrm{T}} \in \mathbb{R}^{q \times q}$. Assume that the system $\mathscr{B}$ represented by Eq. (15) is controllable, and that the representation given by Eq. (15) is trim. Then the following two statements are equivalent:

(1) For all $w \in \mathscr{B} \cap D\left(\mathbb{R}, \mathbb{R}^{q}\right)$,

$\int_{-\infty}^{\infty} w^{\mathrm{T}}(t) M w(t) \mathrm{d} t \geqslant 0$

(2) There exists a symmetric matrix $P \in \mathbb{R}^{p \times p}$ such that

$M+F^{\mathrm{T}} P G+G^{\mathrm{T}} P F \geqslant 0$.

Proof.

$\forall w \in \mathscr{B} \cap D\left(\mathbb{R}, \mathbb{R}^{q}\right): \int_{-\infty}^{\infty} w^{\mathrm{T}}(t) M w(t) \mathrm{d} t \geqslant 0$

$\Uparrow\{$ Lemma 2.2$\}$

$\exists \Psi \in \mathbb{B}_{m}^{q \times q}[\zeta, \eta]$ s.t.

$\forall w \in \mathscr{B}: \frac{\mathrm{d}}{\mathrm{d} t}\left(Q_{\Psi}(w)\right) \leqslant w^{\mathrm{T}} M w$

$\mathbb{\Downarrow}\{G w$ is a state variable, Lemma 2.3$\}$

$\exists E=E^{\mathrm{T}} \in \mathbb{R}^{p \times p}$ s.t.

$\forall w \in \mathscr{B}: \frac{\mathrm{d}}{\mathrm{d} t}\left(w^{\mathrm{T}} G^{\mathrm{T}} E G w\right) \leqslant w^{\mathrm{T}} M w$

$\mathbb{1}\{P=-E\}$

$\exists P=P^{\mathrm{T}} \in \mathbb{R}^{p \times p}$ s.t.

$\forall w \in \mathscr{B}:-\frac{\mathrm{d}}{\mathrm{d} t}\left(w^{\mathrm{T}} G^{\mathrm{T}} P G w\right) \leqslant w^{\mathrm{T}} M w$ 


$$
\begin{aligned}
& \mathbb{1}\{\text { product rule }\} \\
& \begin{aligned}
\exists P= & P^{\mathrm{T}} \in \mathbb{R}^{p \times p} \text { s.t. } \\
& \forall w \in \mathscr{B}: w^{\mathrm{T}}\left(M+F^{\mathrm{T}} P G+G^{\mathrm{T}} P F\right) w \geqslant 0
\end{aligned} \\
& \mathbb{i}\{\text { the representation given by Eq. }(15) \text { is trim }\} \\
& \exists P=P^{\mathrm{T}} \in \mathbb{R}^{p \times p} \text { s.t. } M+F^{\mathrm{T}} P G+G^{\mathrm{T}} P F \geqslant 0 .
\end{aligned}
$$

Remark. Lemma 3.1 is slightly different from the result in [1], where it is not assumed that the representation given by Eq. (15) is trim, but where the matrix $G$ is assumed to have full row-rank. Note that if the system $\mathscr{B}$ is controllable, then the representation given by Eq. (15) is trim if the matrix $G$ has full row-rank.

\section{The KYP Lemma for higher-order systems}

We start off this section with a controllable system in kernel representation, and in a few steps we transform it into a generalized first-order system that is controllable and trim. The first-order representation is formulated in terms of the original coefficients of the higher-order system. Consider the system $\mathscr{B}$ with kernel representation

$R\left(\frac{\mathrm{d}}{\mathrm{d} t}\right) w=0$

Here $R \in \mathbb{R}^{p \times q}[\xi]$ is a $p \times q$ polynomial matrix of degree $N$,

$$
R(\xi)=R_{0}+R_{1} \xi+R_{2} \xi^{2}+\cdots+R_{N} \xi^{N}
$$

and $w$ is the manifest variable. Let $r_{i}$ denote the $i$ th row of $R$, and let $\rho_{i} \leqslant N$ denote its row degree, that is, the maximum of the degrees of the entries in row $r_{i}$.

Step 1: The first transformation step is to build an 'extended' kernel representation by differentiating some of the constraints. Consider the following polynomial matrix containing the rows and some derivatives of the rows of $R$ :

$$
R^{\mathrm{e}}(\xi):=\left(\begin{array}{c}
R_{1}^{\mathrm{e}}(\xi) \\
R_{2}^{\mathrm{e}}(\xi) \\
\vdots \\
R_{p}^{\mathrm{e}}(\xi)
\end{array}\right),
$$

where

$$
R_{i}^{\mathrm{e}}(\xi):=\left(\begin{array}{c}
r_{i}(\xi) \\
\xi r_{i}(\xi) \\
\vdots \\
\xi^{N-\rho_{i}} r_{i}(\xi)
\end{array}\right) .
$$

Note that the 'extended' kernel representation

$R^{\mathrm{e}}\left(\frac{\mathrm{d}}{\mathrm{d} t}\right) w=0$

describes the same behaviour as Eq. (26).

Step 2: The second transformation step is to put the variable $w$ and all its relevant derivatives into a vector $\ell$,

$\ell:=\left(\begin{array}{c}w \\ \dot{w} \\ \ddot{w} \\ \vdots \\ w^{(N)}\end{array}\right)$

This enables us to derive a first-order latent variable representation with external variable $w$ and latent variable $\ell$.

$\left(I_{N q} 0\right) \dot{\ell}=\left(\begin{array}{ll}0 I_{N q}\end{array}\right) \ell$,

$\widetilde{R^{\mathrm{e}} \ell}=0$,

$w=\left(\begin{array}{ll}I_{q} & 0\end{array}\right) \ell$.

Here, for given $n, I_{n}$ denotes the $n \times n$ identity matrix, and $\widetilde{R^{\mathrm{e}}}$ denotes the coefficient matrix of $R^{\mathrm{e}}$. Note that $\widetilde{R^{c}}$ has dimensions $\left(\sum_{i=1}^{p} N+1-\rho_{i}\right)$ by $(N+1) q$. The (manifest) behaviour of this latent variable representation is

$$
\begin{array}{r}
\mathscr{B}_{\ell \mathrm{v}}:=\left\{w \in \mathscr{C}^{\infty}\left(\mathbb{R}, \mathbb{R}^{q}\right) \mid \exists \ell \in \mathscr{C}^{\infty}\left(\mathbb{R}, \mathbb{R}^{(N+1) q}\right)\right. \\
\text { s.t. Eqs. (32)-(34)\}. }
\end{array}
$$

Note that $\mathscr{B}_{\ell \mathrm{v}}=\mathscr{B}$, that is, this latent variable representation still describes the same behaviour.

Step 3: The final transformation step is to eliminate the algebraic constraint given by Eq. (33) by rewriting it in image representation

$\widetilde{R^{\mathrm{e}} \ell}=0 \Leftrightarrow \ell={\widetilde{\mathrm{R}^{\mathrm{e}}}}^{\perp} m$

for some vector $m$ of dimension $d=(N+1) q-$ $\sum_{i=1}^{p} N+1-\rho_{i}$, where ${\widetilde{R^{\mathrm{e}}}}^{\perp}$ is any matrix with $d$ columns such that

$\operatorname{ker}\left(\widetilde{R^{\mathrm{e}}}\right)=\operatorname{image}\left({\widetilde{R^{\mathrm{e}}}}^{\perp}\right)$. 
This induces the following generalized first-order representation with external variable $w$ and latent variable $m$ :

$\left(\begin{array}{ll}I_{N q} & 0\end{array}\right){\widetilde{R^{\mathrm{e}}}}^{\perp} \dot{m}=\left(\begin{array}{ll}\left(I_{N q}\right.\end{array}\right){\widetilde{R^{\mathrm{e}}}}^{\perp} m$,

$w=\left(\begin{array}{ll}I_{q} & 0\end{array}\right) \widetilde{R}^{\perp} m$.

The (manifest) behaviour of this generalized first-order representation is

$$
\begin{array}{r}
\mathscr{B}_{\text {gfo }}:=\left\{w \in \mathscr{C}^{\infty}\left(\mathbb{K}_{3}, \mathbb{R}^{q}\right) \mid \exists m \in \mathscr{C}^{\infty}\left(\mathbb{R}, \mathbb{R}^{d}\right)\right. \\
\text { s.t. Eqs. (38) and (39) }\} .
\end{array}
$$

Note that this representation describes the same behaviour as the kernel representation that we started with: $\mathscr{B}=\mathscr{B}_{\text {gfo }}$.

We must ensure that the representation given by Eq. (38) is trim before we can plug it into the KYP Lemma for generalized first-order systems.

Lemma 4.1. Assume that the matrix $R$ is row-reduced. Then the generalized first-order representation given by Eq. (38) is trim.

Proof. See Appendix A.

A polynomial matrix of full row-rank can be made row-reduced by premultiplication with a suitable unimodular matrix, see for instance [2]. Therefore, we may assume without loss of generality, that a kernel representation is rovi-reduced. The main result of this section is now formulated as follows

Theorem 4.2. Let $\Phi(\zeta, \eta)$ be a symmetric twovariable polynomial matrix as in Eq. (5), with $K \leqslant N$. Assume that the system $\mathscr{B}$ represented by $E q$. (26) is controllable, and that the matrix $R$ is row-reduced. Then the following two statements are equivalent:

(1) For all $w \in \mathscr{B} \cap \cap D\left(\mathbb{R}, \mathbb{R}^{q}\right)$,

$\int_{-\infty}^{\infty} Q_{\Phi}(w)(t) \mathrm{d} t \geqslant 0$

(2) There exists a symmetric matrix $P \in \mathbb{R}^{N q \times N q}$ such that

$$
\begin{aligned}
\widetilde{R}^{\mathrm{e}^{\perp \mathrm{T}}}(\tilde{\Phi} & +\left(\begin{array}{cc}
0_{N q \times q} & P \\
0_{q \times \xi^{\prime}} & 0_{q \times N q}
\end{array}\right) \\
& \left.+\left(\begin{array}{cc}
0_{q \times N q} & 0_{q \times q} \\
P & 0_{N q \times q}
\end{array}\right)\right) \widetilde{R}^{\perp} \geqslant 0 .
\end{aligned}
$$

Proof. Since $\mathscr{B}=\mathscr{B}_{\text {gfo }}$, Eq. (41) is equivalent to saying that for all $m \in D\left(\mathbb{R}, \mathbb{R}^{d}\right)$ that satisfy Eq. (38),

$$
\int_{-\infty}^{\infty} m^{\mathrm{T}}(t){\widetilde{R^{\mathrm{e}}}}^{\perp \mathrm{T}} \tilde{\Phi} \widetilde{R}^{\mathrm{e}^{\perp}} m(t) \mathrm{d} t \geqslant 0 .
$$

Moreover, since $R$ is row-reduced, the representation given by Eq. (38) is trim by Lemma 4.1. The result follows using Lemma 3.1.

Remark 4.3. There is a one-one relationship between solutions $P$ to the linear matrix inequality given by Eq. (42) and storage functions $Q_{\Psi}$ for $Q_{\Phi}$ on $\mathscr{B}$, defined by

$\tilde{\Psi}=-P$.

Consequently, these storage functions are of the form

$$
\begin{aligned}
Q_{\Psi}(w) & =\ell^{\mathrm{T}}\left(\begin{array}{cc}
\tilde{\Psi} & 0_{N q \times q} \\
0_{q \times N q} & 0_{q \times q}
\end{array}\right) \ell \\
& =-\ell^{\mathrm{T}}\left(\begin{array}{cc}
P & 0_{N q \times q} \\
0_{q \times N q} & 0_{q \times q}
\end{array}\right) \ell .
\end{aligned}
$$

Remark. It is still possible to use Theorem 4.2 even if the degree $K$ of $\Phi$ is larger than $N$. In this case the degree of the matrix $R$ must first be increased artificially by imposing $R_{N+1}=R_{N+2}=\cdots=R_{K}=$ : $0_{p \times q}$.

\section{Polynomial spectral factorization}

In this last section we present a new algorithm for polynomial spectral factorization. This algorithm is based on the use of a suitable LMI. We begin by introducing some results from factorization theory. A square polynomial matrix $M$ is para-Hermitian if $M^{\mathrm{T}}(-\xi)=M(\xi)$. It may be found in for instance [3], that if a para-Hermitian matrix $M$ satisfies

$M(i \omega) \geqslant 0$ for all $\omega \in \mathbb{R}$,

then there exist square polynomial matrices $F(\xi)$ and $C(\xi)$ such that

$M(\xi)=F^{\mathrm{T}}(-\xi) F(\xi)$

and

$M(\xi)=C(\xi) C^{\mathrm{T}}(-\xi)$.

These factorizations are called a symmetric polynomial spectral factorization and spectral co-factorization 
of $M(\xi)$, respectively. Moreover, if

$M(\mathrm{i} \omega)>0$ for all $\omega \in \mathbb{R}$,

then $M$ admits a Hurwitz factorization and cofactorization,

$M(\xi)=F_{\mathrm{H}}^{\mathrm{T}}(-\check{\zeta}) F_{\mathrm{H}}(\xi)$

and

$M(\xi)=C_{\mathrm{H}}(\xi) C_{\mathrm{H}}^{\mathrm{T}}(-\xi), \quad$ respectively,

where $F_{\mathrm{H}}$ and $C_{\mathrm{H}}$ are square and Hurwitz ${ }^{1}$ polynomial matrices.

Consider now a symmetric two-variable polynomial matrix $\Phi$ that satisfies

$\Phi(-i \omega, i \omega)>0$ for all $\omega \in \mathbb{R}$.

Our new spectral factorization technique is to derive an LMI for finding a square and Hurwitz spectral factor $F_{\mathrm{H}}(\xi)$ and co-factor $C_{\mathrm{H}}(\xi)$ such that

$\Phi(-\xi, \xi)=F_{\mathrm{H}}^{\mathrm{T}}(-\xi) F_{\mathrm{H}}(\xi)=C_{\mathrm{H}}(\xi) C_{\mathrm{H}}^{\mathrm{T}}(-\xi)$.

Note that this also solves the factorization problem for an arbitrary square, para-Hermitian polynomial matrix $M \in \mathbb{R}^{q \times q}[\xi]$ that satisfies Eq. (50), since it is always possible to transform $M$ into $\Phi$ by taking

$\Phi(\zeta, \eta):=\frac{1}{2}\left(M^{\mathrm{T}}(\zeta)+M(\eta)\right)$.

Indeed we have $\Phi(-\xi, \xi)=M(\xi)$, since $M(\xi)$ is para-Hermitian.

The following result from [8] gives a characterization of dissipativity in terms of non-negativity of $\Phi(-\xi, \xi)$ along the imaginary axis.

\section{Lemma 5.1.}

$\int_{-\infty}^{\infty} Q_{\Phi}(w)(t) \mathrm{d} t \geqslant 0 \quad$ for all $w \in D\left(\mathbb{R}, \mathbb{R}^{q}\right)$

$\Leftrightarrow \Phi(-\mathrm{i} \omega, \mathrm{i} \omega) \geqslant 0 \quad$ for all $\omega \in \mathbb{R}$.

This lemma is used in [8] to derive the following result that relates storage functions to spectral factorizations.

Lemma 5.2. (1) Assume that

$\int_{-\infty}^{\infty} Q_{\Phi}(w)(t) \mathrm{d} t \geqslant 0 \quad$ for all $w \in D\left(\mathbb{R}, \mathbb{R}^{q}\right)$.

\footnotetext{
${ }^{1}$ A square polynomial matrix $H$ is Hurwitz if the real parts of the roots of $\operatorname{det}(H)$ are negative.
}

Then there exist storage functions $Q_{\Psi-}$ and $Q_{\Psi^{+}}$for $Q_{\Phi}$ such that

$Q_{\Psi^{-}} \leqslant Q_{\Psi} \leqslant Q_{\Psi^{+}}$

for every storage function $Q_{\Psi}$ for $Q_{\Phi}$.

(2) Assume that $\Phi(-\mathrm{i} \omega, \mathrm{i} \omega)>0$ for all $\omega \in \mathbb{R}$. Let $F_{\mathrm{H}}(\xi)$ and $C_{\mathrm{H}}(\xi)$ be a square and Hurwitz spectral factor and co-factor of $\Phi(-\xi, \xi)$, respectively. Then

$\Psi^{-}(\zeta, \eta)=\frac{\Phi(\zeta, \eta)-F_{\mathrm{H}}^{\mathrm{T}}(\zeta) F_{\mathrm{H}}(\eta)}{\zeta+\eta}$

and

$\Psi^{+}(\zeta, \eta)=\frac{\Phi(\zeta, \eta)-C_{\mathrm{H}}(\eta) C_{\mathrm{H}}^{\mathrm{T}}(\zeta)}{\zeta+\eta}$

The following theorem is our result on polynomial spectral factorization.

Theorem 5.3. Let $\Phi(\zeta, \eta)$ be a symmetric two-variable polynomial matrix of degree $K$, as in Eq. (5). Consider the following linear matrix inequality in the symmetric $\mathrm{Kq} \times \mathrm{Kq}$ matrix $P$ :

$\tilde{\Phi}+\left(\begin{array}{cc}0_{K q \times q} & P \\ 0_{q \times q} & 0_{q \times K q}\end{array}\right)+\left(\begin{array}{cc}0_{q \times K q} & 0_{q \times q} \\ P & 0_{K q \times q}\end{array}\right) \geqslant 0$.

Let $F(P)$ denote the left-hand side of the inequality given by Eq. (62), and define the solution set of Eq. (62) as $\mathscr{S}:=\left\{P=P^{\mathrm{T}} \in \mathbb{R}^{K q \times K q}\right.$ s.t. Eq. (62) $\}$.

Assume that $\Phi$ satisfies

$\Phi(-\mathrm{i} \omega, \mathrm{i} \omega)>0$ for all $\omega \in \mathbb{R}$.

Then $\mathscr{S}$ is non-empty. Moreover, there exist $P^{-}$, $P^{+} \in \mathscr{S}$ such that $P^{-} \leqslant P \leqslant P^{+}$for all $P \in \mathscr{S}$. Furthermore,

$\operatorname{rank}\left(F\left(P^{-}\right)\right)=\operatorname{rank}\left(F\left(P^{+}\right)\right)=q$.

Consequently, there exist $\tilde{V} \in \mathbb{R}^{q \times(K+1) q}$ and $\tilde{Q} \in$ $\mathbb{R}^{(K+1) q \times q}$ such that

$F\left(P^{+}\right)=\tilde{V}^{\mathrm{T}} \tilde{V} \quad$ and $\quad F\left(P^{-}\right)=\tilde{Q} \tilde{Q}^{\mathrm{T}}$.

Define now

$V(\xi):=\tilde{V}\left(\begin{array}{c}I \\ \xi I \\ \vdots \\ \xi^{K} I\end{array}\right)$ 
and

$Q(\xi):=\left(\begin{array}{llll}I \quad \xi I & \cdots & \xi K\end{array}\right) \tilde{Q}$.

Then $V(\xi)$ and $Q(\xi)$ are a square and Hurwitz factor and co-factor of $\Phi(-\xi, \xi)$, respectively.

Proof. By Lemma 5.1, condition given by Eq. (63) implies that for all $v \in D\left(\mathbb{R}, \mathbb{R}^{q}\right)$,

$$
\int_{-\infty}^{\infty} Q_{\Phi}(w)(t) \mathrm{d} t \geqslant 0 .
$$

By Theorem 4.2, Eq. (67) implies that $\mathscr{S} \neq \emptyset$. Moreover, by Remark 4.3, $Q_{\Psi}$ is a storage function for $Q_{\Phi}$ for all $\Psi$ such that $-\tilde{\Psi}=P \in \mathscr{S}$. It follows from Lemma 5.2(1) that there exist $P^{-}, P^{+} \in \mathscr{S}$ such that $P^{-} \leqslant P \leqslant P^{+}$for all $P \in \mathscr{S}$. In fact, $\tilde{\Psi}^{-}=-P^{+}$and $\tilde{\Psi}^{+}=-P^{-}$. Moreover,

$$
\begin{aligned}
& \left(\begin{array}{c}
I \\
\zeta I \\
\vdots \\
\zeta^{K} I
\end{array}\right)^{\mathrm{T}} F\left(P^{-}\right)\left(\begin{array}{c}
I \\
\eta I \\
\vdots \\
\eta^{K} I
\end{array}\right) \\
& \quad=\Phi(\zeta, \eta)-(\zeta+\eta) \Psi^{+}(\zeta, \eta)
\end{aligned}
$$

and

$$
\begin{aligned}
& \left(\begin{array}{c}
I \\
\zeta I \\
\vdots \\
\zeta^{K} I
\end{array}\right)^{\mathrm{T}} F\left(P^{+}\right)\left(\begin{array}{c}
I \\
\eta I \\
\vdots \\
\eta^{K} I
\end{array}\right) \\
& =\Phi(\zeta, \eta)-(\zeta+\eta) \Psi^{-}(\zeta, \eta) .
\end{aligned}
$$

Let $F_{\mathrm{H}}(\xi)$ and $C_{\mathrm{H}}(\xi)$ be a square and Hurwitz spectral factor and co-factor of $\Phi(-\xi, \xi)$, respectively. Then by Lemma 5.2(2), the expressions in Eqs. (68) and (69) are equal to $F_{\mathrm{H}}^{\mathrm{T}}(\zeta) F_{\mathrm{H}}(\eta)$ and $C_{\mathrm{H}}(\eta) C_{\mathrm{H}}^{\mathrm{T}}(\zeta)$, respectively. This implies that

$\operatorname{rank}\left(F\left(P^{-}\right)\right) \leqslant q$ and $\operatorname{rank}\left(F\left(P^{+}\right)\right) \leqslant q$.

Condition (63) implies that $\tilde{\Phi}_{00}=\Phi(0,0)>0$, so that

$\operatorname{rank}(F(P)) \geqslant q$ for all $P \in \mathscr{S}$.

It follows that

$\operatorname{rank}\left(F\left(P^{-}\right)\right)=\operatorname{rank}\left(F\left(P^{+}\right)\right)=q$.

Again by Lemma 5.2(2), for any choice of $\tilde{V}$ and $\tilde{Q}$, $V^{\mathrm{T}}(\zeta) V(\eta)=F_{\mathrm{H}}^{\mathrm{T}}(\zeta) F_{\mathrm{H}}(\eta)$

and

$Q(\eta) Q^{\mathrm{T}}(\zeta)=C_{\mathrm{H}}(\eta) C_{\mathrm{H}}^{\mathrm{T}}(\zeta)$,
This is equivalent to saying that

$$
\left\|V\left(\frac{\mathrm{d}}{\mathrm{d} t}\right) w\right\|^{2}=\left\|F_{\mathrm{H}}\left(\frac{\mathrm{d}}{\mathrm{d} t}\right) w\right\|^{2}
$$

and

$$
\left\|Q\left(\frac{\mathrm{d}}{\mathrm{d} t}\right) w\right\|^{2}=\left\|C_{\mathrm{H}}\left(\frac{\mathrm{d}}{\mathrm{d} t}\right) w\right\|^{2} .
$$

This implies that $V(\xi)$ and $Q(\xi)$ are also a square and Hurwitz factor and co-factor of $\Phi(-\xi, \xi)$, respectively.

\section{Appendix A: Proof of Lemma 4.1}

We prove that if $R$ is row-reduced, then for all $\ell_{0} \in \operatorname{ker}\left(\widetilde{R^{\mathrm{e}}}\right)$ there exists an $\ell(t) \in \operatorname{ker}\left(\widetilde{R^{\mathrm{e}}}\right)$ that satisfies Eq. (32) and $\ell(0)=\ell_{0}$. This proves Lemma 4.1 because of the relation between $\ell$ and $m$.

Note that

$\widetilde{R_{i}^{\mathrm{e}}}=\left(\begin{array}{c}\widetilde{R_{i 1}^{\mathrm{e}}} \\ \widetilde{R_{i 2}^{\mathrm{e}}} \\ \vdots \\ \widetilde{R_{i \rho_{i}}^{\mathrm{e}}}\end{array}\right)$,

where

$\widetilde{R_{i j}^{\mathrm{e}}}:=\left(0_{1 \times q(j-1)} \tilde{r}_{i} 0_{1 \times q\left(\rho_{i}-j\right)}\right)$.

Consider the matrix $\widetilde{R_{\mathrm{L}}^{\mathrm{e}}}$ containing the last rows of $\widetilde{R_{i}^{\mathrm{e}}}$ for $i=1, \ldots, p$,

$\widetilde{R_{\mathrm{L}}^{\mathrm{e}}}:=\left(\begin{array}{c}\widetilde{R_{1}^{\mathrm{e}}} \rho_{1} \\ \widetilde{R_{2}^{\mathrm{e}} \rho_{2}} \\ \vdots \\ \widetilde{R_{p}^{\mathrm{e}}}\end{array}\right)$.

Partition $\widetilde{R_{\mathrm{L}}^{\mathrm{e}}}$ as follows:

$\widetilde{R_{\mathrm{L}}^{\mathrm{e}}}=:\left(\begin{array}{ll}\widetilde{R_{\mathrm{L} 1}^{\mathrm{e}}} & \underset{q \text { columns }}{\widetilde{R_{\mathrm{L} 2}^{c}}}\end{array}\right)$.

Note that $\widetilde{R_{\mathrm{L} 2}^{\mathrm{e}}}$ has full row-rank, since $R$ is row-reduced. Let ${\widetilde{R_{\mathrm{L} 2}^{\mathrm{e}}}}^{\dagger}$ denote a right-inverse of $\widetilde{R_{\mathrm{L} 2}^{\mathrm{e}}}$.

Now pick an arbitrary $\ell_{0} \in \operatorname{ker}\left(\widetilde{R^{\mathrm{e}}}\right)$ and partition it in line with the partition in Eq. (A.4),

$\ell_{0}=:\left(\begin{array}{l}\ell_{01} \\ \ell_{02}\end{array}\right)$. 
The signal $\ell(t)$ has the same partition,

$\ell(t)=:\left(\begin{array}{l}\ell_{1}(t) \\ \ell_{2}(t)\end{array}\right)$

Define $\ell_{1}(t)$ as the solution to the differential equation

$\dot{\ell}_{1}(t)=\left(\frac{0_{(N-1) q \times q} \mid I_{(N-1) q}}{-{\widetilde{R_{\mathrm{L} 2}^{\mathrm{e}}}}^{\dagger} \widetilde{R_{\mathrm{L} 1}^{\mathrm{e}}}}\right) \ell_{1}(t)$,

with initial conditions $\ell_{1}(0):=\ell_{10}$. Define $\ell_{2}(t)$ as

$\ell_{2}(t):=-{\widetilde{R_{\mathrm{L}}^{\mathrm{e}}}}^{\dagger} \widetilde{R_{\mathrm{L} 1}^{\mathrm{e}}} \ell_{1}(t)$.

This choice of $\ell(t)$ satisfies Eq. (32) and $\ell(0)=\ell_{0}$. In addition,

$\widetilde{R_{\mathrm{L}}^{\mathrm{e}}} \ell(t)=0$ for all $t$.

Note that since $\ell_{0} \in \operatorname{ker}\left(\widetilde{R^{\mathrm{e}}}\right)$,

$\widetilde{R_{i p_{i}}^{\mathrm{e}}-1} \ell(0)=0$.

From Eqs. (32) and (A.9) it follows that

$\widetilde{R_{i \rho_{i}}^{\mathrm{e}}-1} \dot{\ell}(t)=\widetilde{R_{i \rho_{i}}^{\mathrm{e}}} \ell(t)=0$ for all $t$.

Eqs. (A.10) and (A.11) together imply that

$\widetilde{R_{i p_{i}}^{\mathrm{e}}-1} \ell(t)=0 \quad$ for all $t$.
By repeating this argument $\rho_{i}-1$ times we prove that $\widetilde{R_{i}^{\mathrm{e}} \ell} \ell(t)=0$. It follows that $\ell(t) \in \operatorname{ker}\left(\widetilde{R^{\mathrm{e}}}\right)$.

\section{References}

[1] R.A.B. van der Geest, A.R. Rantzer, Quadratic performance of generalized first-order systems, Proc. 35th IEEE CDC, Kobe, Japan, 1996.

[2] T. Kailath, Linear Systems, Prentice-Hall, Englewood Cliffs, NJ, 1980.

[3] H. Kwakernaak, M. Šbek, Polynomial $J$-spectral factorization, IEEE Trans. Automat. Control 39 (1994) 315-328.

[4] P. Rapisarda, J.C. Willems, State maps for linear systems, SIAM J. Control Optim. 1995, accepted.

[5] H.L. Trentelman, J.C. Willems, Every storage function is a state function, Systems Control Lett. 32 (1997) 249-259, this issue.

[6] V.A. Yakubovich, A frequency domain theorem in control theory, translated from Sibirskii Matematicheskii Zhurnal 14 (2) (1962) 384-419.

[7] J.C. Willems, Paradigms and puzzles in the theory of dynamical systems, IEEE Trans. Automat. Control 36 (1991) 259-294.

[8] J.C. Willems, H.L. Trentelman, On quadratic differential forms, SIAM J. Control Optim., accepted. 